Pacific Journal of Mathematic 


\title{
A CHARACTERIZATION OF THE TOPOLOGY OF COMPACT CONVERGENCE ON $C(X)$
}

\author{
William A. Feldman
}

The function space of all continuous real-valued functions on a realcompact topological space $X$ is denoted by $C(X)$. It is shown that a topology $\tau$ on $C(X)$ is a topology of uniform convergence on a collection of compact subsets of $X$ if and only if $\left.{ }^{*}\right) C_{\tau}(X)$ is a locally $m$-convex algebra and a topological vector lattice. Thus, the topology of compact convergence on $C(X)$ is characterized as the finest topology satisfying $\left(^{*}\right)$. It is also established that if $C_{\tau}(X)$ is an $A$-convex algebra (a generalization of locally $m$-convex) and a topological vector lattice, then each closed (algebra) ideal in $C_{\tau}(X)$ consists of all functions vanishing on a fixed subset of $X$. Some consequences for convergence structures are investigated.

Introduction. Throughout this paper, $X$ will denote a realcompact topological space and $C(X)$ the algebra and lattice of all realvalued continuous functions on $X$ under the pointwise defined operations. After preliminary remarks in $\S 1$, we describe (Theorem 1) closed (algebra) ideals in $C(X)$ endowed with a topology $\tau$ making $C_{\tau}(X)$ an $A$-convex algebra (a generalization of locally $m$-convex introduced in [4]) and a topological vector lattice. As a corollary, we state sufficient conditions for $\tau$ so that an ideal in $C_{\tau}(X)$ is closed if and only if it consists of all functions vanishing on a subset of $X$. Then, in Theorem 3, we characterize topologies on $C(X)$, which are topologies of uniform convergence on a collection of compact subsets of $X$. In particular, the corollary of Theorem 3 provides a characterization of the topology of compact convergence on $C(X)$. We conclude the note $(\S 3)$, by discussing generalizations applicable to convergence structures on $C(X)$.

1. Definitions and preliminary results. Since our major concern is the algebra $C(X)$, we restrict our definitions to commutative algebras over the reals.

Definition 1. Given a commutative $R$-algebra $\mathscr{A}$, an absolutely convex subset $S \subset \mathscr{A}$ is said to be m-convex (respectively, $A$-convex) if $S \cdot S=\{f g: f, g \in S\}$ is contained in $S$ (respectively, $f S=\{f g: g \in S\}$ is absorbed by $S$ for each $f$ in $S$ ). Now $(\mathscr{A}, \tau)$, the algebra $\mathscr{A}$ together with a convergence structure $\tau$ (see [1]) is said to be an $m$-convex (respectively, A-convex) convergence algebra if $\tau$ is a convergence vector space structure (see [1]) and for every filter $\theta$ con- 
vergent to zero (in $(\mathscr{A}, \tau)$ ), there exists a coarser filter $\Phi$ convergent to zero with a basis consisting of $m$-convex (respectively, $A$-convex) sets.

It is evident that if $\tau$ is a topology, these definitions coincide with the concepts of a locally $m$-convex algebra (respectively, an $A$-convex algebra as defined in [4]). Since every $m$-convex set is $A$-convex, every $m$-convex convergence algebra is also an $A$-convex convergence algebra.

DeFINITION 2. In a vector lattice $L$, a subset $S$ of $L$ is said to be solid, if $f \subset S$ whenever $|f| \leqq|g|(f \in L)$ and $g \in S$ (e.g., see [8], p. 35). Given a convergence vector space structure $\tau$ on $L$ with the property that for every filter $\theta$ convergent to zero, there exists a coarser filter $\Phi$ convergent to zero, where $\Phi$ has a basis of solid sets, we call $(L, \tau)$ a convergence vector lattice.

Clearly, a convergence vector lattice that is also a topological space is a topological vector lattice. Further, one can readily verify that in every convergence vector lattice, the lattice operations are continuous.

The algebra $C(X)$ is a lattice with respect to the order induced by the cone of nonnegative functions. Thus for $f \in C(X)$, the function $|f|$ may be characterized by $|f|(x)=|f(x)|$ for every $x \in X$. The symbols " $\vee$ " and " $\wedge$ " will denote the lattice operations of "sup" "inf", respectively. In addition, we will use the notation " 1 " to represent the function of constant value 1 .

Definition 3. Let $C_{\tau}(X)$ denote $C(X)$ together with the convergence structure $\tau$. Now, the space $C_{\tau}(X)$ will be called an $m$ convex (respectively, A-convex) convergence lattice if it is both an $m$ convex (respectively, $A$-convex) convergence algebra and a convergence vector lattice with respect to the natural order defined above. If, in addition, $C_{\tau}(X)$ is a topological space, we will substitute the word "topological" for "convergence".

In discussing $A$-convex convergence lattices, the following two technical results will prove important.

Lemma 1. Let $C_{\tau}(X)$ be an A-convex convergence lattice. For every filter $\theta$ convergent to zero in $C_{\tau}(X)$, there exists a coarser filter $\Phi$ convergent to zero, where $\Phi$ has a basis consisting of solid, A-convex sets. If, in addition $\tau$ is a topology, members of a basis for $\Phi$ can be chosen closed, A-convex and solid.

Proof. Let $\theta$ be a filter convergent to zero in $C_{\tau}(X)$. Since the Fréchet filter $\mathscr{F}$ generated by $\left(\frac{1}{n} 1\right)_{n \in N}$ converges to zero, the as- 
sumption implies that there exists a filter $\psi$ coarser than $\mathscr{F} \cap \theta$ and having a basis of solid sets. In turn, $\psi$ is finer than a filter $\Omega$ having a basis $\mathscr{B}$ consisting of $A$-convex sets. For each $B \in \mathscr{B}$, we define $B^{\prime}$ to be the collection of all functions $f \in B$ with the property that if $|g| \leqq|f|(g \in C(X))$ then $g \in B$. Clearly, $B^{\prime}$ is solid and since $B$ contains a solid set $S$ in $\psi$, we have $B^{\prime} \supset S$ (i.e., $B^{\prime} \in \psi^{\prime}$ ). Moreover, we will show that $B^{\prime}$ is an $A$-convex set. First, to verify that $B^{\prime}$ is absolutely convex, let $f, g$ be in $B^{\prime}$ and $k$ any function in $C(X)$ with $|k| \leqq|\lambda f+\beta g|$, where $(|\lambda|+|\beta|) \leqq 1$. We need only verify that $k \in B$. Now the function

$$
h=(k \wedge|\lambda \| f|) \vee(-|\lambda \| f|)
$$

has the property that $|h| \leqq|\lambda||f|$ and $|k-h| \leqq|\beta||g|$. Since $f$ and $g$ are elements of $B^{\prime}$, both $h / \lambda$ and $(k-h) / \beta$ are in $B$. The absolute convexity of $B$ implies $k$ is in $B$. Now, to show that $B^{\prime}$ is $A$-convex, let $g$ be any member of $B^{\prime}$. Since $\Omega$ is contained in $\mathscr{F}$, the function 1 is absorbed by $B^{\prime}$ and hence, $|g|+1$ is absorbed by $B^{\prime}$. We know that $B^{\prime}$ is contained in the $A$-convex set $B$, which implies that for some $\lambda>0$,

$$
\left(^{*}\right)(|g|+1) \cdot B^{\prime} \subset \alpha B \text { for every } \alpha \geqq \lambda .
$$

We wish to demonstrate that $g B^{\prime} \subset \alpha B^{\prime}$ for $\alpha \geqq \lambda$. Given $k \in C(X)$ with $|k| \leqq|g f|$ and $f$ an element of $B^{\prime}$, it suffices to show that $k \in \lambda B$. Clearly, $|k| /(|g|+1)$ is less than or equal to $|f|$, and thus $k /(|g|+1)$ is in $B^{\prime}$. It follows from $\left(^{*}\right)$ that $(|g|+1) k /(|g|+1)$ is in $\lambda B$ as desired. It is evident that $\left(B_{1} \cap B_{2}\right)^{\prime} \subset\left(B_{1}^{\prime} \cap B_{2}^{\prime}\right)$ for $B_{1}, B_{2} \in \mathscr{B}$, and thus $\left\{B^{\prime}: B \in \mathscr{B}\right\}$ is a basis for a filter $\Phi$ convergent to zero since $\Phi \supset \Omega$. We conclude that $\Phi$ has the required properties. Finally, if $C_{\tau}(X)$ is a topological space, it can be easily verified that the closures of neighborhoods $B^{\prime}$ remain $A$-convex and solid.

Any function $f \in C(X)$ can be regarded as a continuous function from $X$ into $\dot{R}$, the one point compactification of the reals. Thus, the function $f$ can be extended (uniquely) to a function $\bar{f}$ from $\beta X$, the Stone-Čech compactification of $X$, into $\dot{R}$. If $f$ is an element of $C^{\circ}(X)$, the collection of all bounded elements in $C(X)$, then $\bar{f}$ can, of course, be regarded as a real-valued function. We will use the concept of a support set as introduced by Nachbin in [7]. Let $V$ be a convex subset of $C^{0}(X)$ (respectively, $C(X)$ ). A compact subset $G \subset$ $\beta X$ with the property that $f$ is an element of $V$ whenever $\bar{f}$ vanishes on $G$ and $f \in C^{\circ}(X)$ (respectively, $C(X)$ ), is called a support set for $V$. The following result for a convex subset of $C(X)$ is due to Nachbin. (See [7].) Its extension to $C^{0}(X)$ is easily obtained from Nachbin's proof. 
Lemma 2. If $V$ is a convex subset of $C^{0}(X)$ (respectively, $C(X)$ ) such that $V \supset\{f:|f| \leqq \delta 1\}$ for some $\delta>0$, then $G(V)$, the intersection of all support sets for $V$, is again a support set for $V$. Further, given any support set $G$ for $V$, if $f \in C^{0}(X)$ (respectively, $f \in C(X)$ ) and

$$
\|f\|_{G}=\sup \{|\bar{f}(x)|: x \in G\}
$$

is less than $\delta / 2$, then $f$ is an element of $V$.

2. Topological algebras. We first prove the following proposition for an $A$-convex topological lattice $C_{\tau}(X)$ to facilitate the study of closed ideals. The symbol $C_{k}^{0}(X)$ will denote the algebra of all bounded elements in $C(X)$ endowed with the topology of convergence.

Proposition 1. If $C_{\tau}(X)$ is an A-convex topological lattice, then the inclusion map from $C_{k}^{0}(X)$ into $C_{\tau}(X)$ is continuous.

Proof. In view of Lemma 1, we can assume that the neighborhood filter of zero in $C_{\tau}(X)$ has a basis, $\mathscr{B}$, consisting of solid $A$-convex sets. Given any element $U$ in $\mathscr{B}$, we will show that $U$ contains a neighborhood of zero in $C_{k}^{0}(X)$. Clearly, $U^{0}=U \cap C^{0}(X)$ is a solid $A$-convex subset of $C^{0}(X)$. Since $U$ is absorbing, $U^{0}$ contains $\left\{f \in C^{0}(X):|f|<\delta 1\right\}$ for some $\delta>0$. Lemma 2 states that

$$
U^{0} \supset\left\{f \in C^{0}(X):\|f\|_{G\left(U^{0}\right)}<\frac{\delta}{2}\right\}
$$

where $G\left(U^{0}\right)$ is the smallest support set for $U^{0}$. Thus, it is sufficient to show that $G\left(U^{0}\right)$ is a subset of $X$. Let $p$ be an arbitrary point in $\beta X \backslash X$. Since $X$ is realcompact, we can choose a function $f \in C(X)$ such that $\bar{f}(p)=\infty$ (for example, the function $1 /|f|$ on page 119 in [5]). For convenience, we can assume $f$ is greater than zero and an element of $U$, since $U$ is absorbing (divide by an appropriate constant). Now $U$ is also $A$-convex so that $f U \subset \alpha U$ for some $\alpha>1$. Letting $k=f / \alpha$, one can verify that $k^{n}$ is in $U$ for every natural number $n$. We will establish that

$$
G=\{x \in \beta X: \bar{k}(x) \leqq 2\}
$$

is actually a support set for $U^{0}$. To this end let $h$ be in $C^{0}(X)$ with $\bar{h}$ vanishing on $G$. There exists a positive integer $m$ so that $|h(x)|<$ $2^{m}$ for every $x \in X$, and thus $|h| \leqq k^{m}$. Since $U$ is solid, $h$ is in both $U$ and $U^{0}$. Hence, $G$ is a support set for $U^{0}$ disjoint from $p$, and it follows from Lemma 2 that $G\left(U^{0}\right)$ is contained in $X$ as desired.

The term ideal will always mean a proper algebra ideal. In $C(X)$ (respectively, in $C^{\circ}(X)$ ), an ideal is said to be full if it consists of all 
functions in $C(X)$ (respectively, in $C^{0}(X)$ ) vanishing on a nonempty subset of $X$. In particular, for $N \subset X$, let $I(N)$ denote the full ideal in $C(X)$ of all functions vanishing on $N$.

Theorem 1. In an A-convex topological lattice $C_{\tau}(X)$, every closed ideal is full.

Proof. Let $J$ be a closed ideal in $C_{\tau}(X)$. Proposition 1 implies that $J^{0}=J \cap C^{0}(X)$ is a closed ideal in $C_{k}^{0}(X)$. Since an ideal is closed in $C_{k}^{\circ}(X)$ if and only if it is full (e.g., this follows from Lemma 3 and Proposition 3 in [2], and the fact that the topology of compact convergence is coarser than the continuous convergence structure), we can write $J^{0}=I(N) \cap C^{0}(X)$, where $N \subset X$. We will show that $J$ is actually the full ideal $I(N)$. Given any function $f \in C(X)$, we have

$$
((-1 \vee f) \wedge 1)=u f \text {, }
$$

where $u$ is a unit (an invertible function) in $C(X)$ (see [5], p. 21). Now if $f \in J$, then $u f$ is an element of $J^{0}$, which implies that $u f$ and hence $f$ vanish on $N$, i.e., $J \subset I(N)$. Conversely, if $f \in I(N)$, we have $u f \in J^{0}$, and finally, $u^{-1} u f=f$ must be an element of $J$. Hence, $J$ is the full ideal $I(N)$.

CoROllary. Let $C_{-}(X)$ be an A-convex topological lattice such that $\tau$ restricted to the bounded functions is finer than the topology of pointwise convergence. An ideal in $C_{\tau}(X)$ is closed if and only if it is full.

Proof. In view of the previous theorem, we need only verify that $I(N)$, for $N \subset X$, is closed in $C_{\tau}(X)$. Assume that $\theta$ is a filter in $C(X)$ convergent to $g$ with a basis in $I(N)$. We define $t(f)$ to be $(-1 \vee f) \wedge 1$ for $f \in C(X)$. Since the lattice operations are continuous, the image filter $t(\theta)$ converges to $g^{\prime}=((-1 \vee g) \wedge 1)$. Furthermore, by assumption $t(\theta) \cap C^{0}(X)$ converges pointwise to $g^{\prime}$, hence, $g$ itself vanishes on $N$. Therefore, $I(N)$ is a closed ideal in $C_{\tau}(X)$.

A seminorm $s$ on $C(X)$ will be called a supremum seminorm, if, for any $f \in C(X)$,

$$
s(f)=\sup \{|f(x)|: x \in K\},
$$

where $K$ is a compact subset of $X$. We say that a topology $\tau$ on $C(X)$ is a topology of k-convergence if $\tau$ is generated by a collection of supremum seminorms, i.e., $\tau$ is a topology of uniform convergence on a collection of compact subsets of $X$.

Before characterizing the topologies of $k$-convergence, we prove the following lemma. 
Lemma 3. Let $C_{\tau}(X)$ be an A-convex topological lattice. Either of the following conditions implies that the sequence $(|f| \wedge n 1)_{n \in N}$ converges to $|f|$ for each $f \in C(X)$ :

(1) $C^{0}(X)$ is dense in $C_{\tau}(X)$.

(2) Inversion is continuous on the set of invertible elements.

Proof. Given that (1) is satisfied and $f$ is any member of $C(X)$, there exists a filter $\theta$ convergent to $|f|$ with a trace on $C^{0}(X)$. This implies that $\theta-|f|$ is finer than a filter $\psi$ convergent to zero with a basis $\mathscr{B}$ of solid sets. Now for any $B \in \mathscr{B}$, there exists an $A \in \theta$ so that $B$ contains $A-|f|$. In particular, $B \supset(g-|f|)$, where $g$ is an element of $A \cap C^{0}(X)$. If $n_{0}$ is a natural number greater than the supremum of $|g|$ on $X$, it follows that

$$
|(|f| \wedge n 1)-| f|| \leqq|g-| f||
$$

for every $n \geqq n_{0}$. Since $B$ is solid, $((|f| \wedge n 1)-|f|)$ is an element of $B$ for every $n \geqq n_{0}$ and we conclude that $(|f| \wedge n 1)_{n \in N}$ converges to $|f|$.

Given that (2) is satisfied, we will show that $C^{0}(X)$ is dense in $C_{-}(X)$, i.e., (1) is satisfied. Let $f$ be a member of $C(X)$ and $U$ any solid neighborhood of zero in $C_{\tau}(X)$. Now there exists a solid neighborhood $V$ of zero with $V+V$ contained in $U$. Proposition 1 implies that there is a $\delta>0$ so that $((|f| \vee \delta 1)-|f|)$ is in $V$. Since inversion is continuous, we can choose a neighborhood $W$ of zero with the property that if $g$ is a unit and $\left(g^{-1}-(|f| \vee \delta 1)^{-1}\right) \in W$, then $(g-$ $(|f| \vee \delta 1))$ is an element of $V$. It is evident that for an appropriate choice of $m>\delta$,

$$
\left[((|f| \vee \delta 1) \wedge m 1)^{-1}-(|f| \vee \delta 1)^{-1}\right] \in W
$$

and thus $[((|f| \vee \delta 1) \wedge m 1)-|f|]$ is in $V+V$. Since $(V+V) \subset U$ and

$$
|((-m 1 \vee f) \wedge m 1)-f| \leqq|((|f| \vee \delta 1) \wedge m 1)-| f||,
$$

we conclude that $C^{0}(X)$ is dense in $C_{\tau}(X)$.

Theorem 2. Let $C_{\tau}(X)$ be an A-convex topological lattice. The topology $\tau$ is coarser than the topology of compact convergence if:

(1) $C^{0}(X)$ is dense in $C_{\tau}(X)$. Or,

(2) Inversion is continuous on the set of invertible elements.

Proof. Let $U$ be any neighborhood of zero in $C_{\tau}(X)$, an $A$-convex topological lattice. In view of Lemma 1 , we may assume $U$ is $A$ convex, solid and closed. Now, Lemma 2 and the fact that $U$ is ab- 
sorbing, implies that $U$ contains $\left\{f \in C(X):\|f\|_{G(U)}<\delta / 2\right\}$, where $\delta>0$ and $G(U)$ is the smallest support set for $U$. To establish the result, we will show (by an argument similar to that in the proof of Proposition 1) that $G(U)$ is contained in $X$ assuming condition (1) or (2) is satisfied. For $p$ any point in $\beta X \backslash X$, choose a positive function $f$ in $U$ with $f(p)=\infty$. We can suppose $f^{n} \in U$ for every $n \in N$ (otherwise, pick an appropriate scalar multiple of $f$ ), and we claim

$$
G=\{x \in \beta X: \bar{f}(x) \leqq 2\}
$$

is a support set for $U$. If $h \in C(X)$ with $\bar{h}(G)=\{0\}$, then $(|h| \wedge n 1) \leqq$ $f^{n}$ for every $n \in N$, and hence, $(|h| \wedge n 1)$ is in $U$. It follows from the supposition and the previous lemma that $h$ itself is in $U$ as desired.

Given that $C_{-}(X)$ is an $m$-convex topological lattice, we know that inversion is continuous (e.g., see [6], Proposition 2.8), and thus, the stipulation of (1) or (2) in the previous theorem can be omitted. Furthermore, we can now prove the following:

THeOREM 3. $C_{i}(X)$ is an m-convex topological lattice, if and only if $\tau$ is a topology of k-convergence.

Proof. Let $C_{\tau}(X)$ be an $m$-convex topological lattice. An $m$ convex closed neighborhood $M$ of zero in $C_{\tau}(X)$ contains a solid closed neighborhood of zero, call it $N$. The proof of Lemma 1 established that the set $M^{\prime}$ consisting of all $f \in M$ with the property that $|g| \leqq$ $|f|$ implies that $g \in M$ is an absolutely convex set containing $N$. We claim that $M^{\prime}$ is also $m$-convex. Given $|k| \leqq|f| \cdot|g|$ for $f, g \in M^{\prime}$, we need only verify that $k$ is in $M$. For any solid neighborhood $U$ of zero, there exists a solid neighborhood $\widetilde{U}$ of zero with $|g| \cdot \widetilde{U} \subset U$. Now, Proposition 1 implies that $\widetilde{U}$ contains $\left\{f \in C^{0}(X):|f| \leqq \delta 1\right\}$ for some $\delta>0$. We have $|k|(|f|+\delta 1)^{-1} \leqq|g|$ since

$$
|k| \leqq|f||g| \leqq(|f|+\delta 1)|g| \text {. }
$$

By writing

$$
\begin{aligned}
k & =\left[k \cdot(|f|+\delta 1)^{-1}\right] \cdot(|f|+\delta 1) \\
& =\left[k \cdot(|f|+\delta 1)^{-1}\right] \cdot|f|+\left[k(|f|+\delta 1)^{-1}\right] \cdot \delta 1,
\end{aligned}
$$

it follows that $k \in(M \cdot M+U)$ and thus $k \in(M+U)$. Since $U$ was arbitrary and $M$ is closed, we conclude that $k$ is indeed in $M$. One may verify that the closure of $M^{\prime}$ is still $m$-convex and solid, and hence, we may choose as a basis for the neighborhoods of zero in $C_{z}(X)$ a collection $\mathscr{l l}$ of closed, $m$-convex, solid sets. For $V \in \mathscr{l l}$, as in the proof of Theorem 2, we have

$$
V \supset\left\{f \in C(X):\|f\|_{G(V)} \leqq \eta\right\}
$$


where $\eta>0$ and $G(V) \subset X$. We will show that

$$
V \subset\left\{f \in C(X):\|f\|_{G(V)} \leqq 3\right\} .
$$

Assume to the contrary that there exists an $f \in V$ with $f(p)>3$ for $p \in G(V)$. Since $V$ is solid, we can assume $f$ is bounded and nonnegative. We will establish that

$$
H=\{x \in \beta X: \bar{f}(x) \leqq 2\}
$$

is a support set for $V$, which will contradict the fact that $G(V)$ is minimal (as $H \cap G(V)$ is a support set). If $g \in C(X)$ with $\bar{g}(H)=\{0\}$, then $(|g| \wedge n 1) \leqq f^{n}$ for every $n \in N$. The fact that $V$ is $m$-convex implies that each $(|g| \wedge n 1)$ is in $V$, and since $V$ is closed and solid, we conclude by an application of Lemma 3 that $g$ is in $V$. Thus, we have proved that $C_{\tau}(X)$ carries a topology of $k$-convergence. The converse is immediate.

COROLlary. The topology of compact convergence on $C(X)$ is the finest among all topologies $\tau$ making $C_{\tau}(X)$ an m-convex topological lattice.

3. Consequences for convergence spaces. Here, we will provide sufficient conditions for closed ideals in $A$-convex convergence lattices to be full.

In studying these structures that are not necessarily topological, we will utilize properties of the Marinescu space $C_{I^{\prime}}(X)$ introduced in [3]. A set $Z \subset \beta X$ is said to be a zero-set if $Z=\{p \in \beta X: f(p)=0\}$ for some function $f$ in $C(\beta X)$. For any zero-set $Z \subset \beta X \backslash X$, the algebra $C(\beta X \backslash Z)$ can be identified with a subalgebra of $C(X)$; namely, restriction of the functions to $X$. The space $C_{I^{\prime}}(X)$ is the inductive limit in the category of convergence spaces of the family

$$
\left\{C_{k}(\beta X \backslash Z): Z \subset \beta X \backslash X \text { is a zero-set }\right\}
$$

together with the order defined by inclusion ( $k$ again denotes the topology of compact convergence). Actually, $C_{I^{\prime}}(X)$ can be regarded as $C(X)$ endowed with the finest convergence structure making all the inclusion maps from members of $\left(^{*}\right)$ into $C(X)$ continuous. Furthermore, it is easily verified that $C_{I^{\prime}}^{0}(X)$, the bounded functions together with the convergence structure inherited from $C_{I^{\prime}}(X)$, coincides with the inductive limit of the family of all $C_{k}^{0}(\beta X \backslash Z)$ for $Z$ a zero-set contained in $\beta X \backslash X$.

In analogy to Proposition 1, we prove:

Proposition 2. If $C_{\tau}(X)$ is an A-convex convergence lattice, then the inclusion map from $C_{I^{\prime}}^{0}(X)$ into $C_{\tau}(X)$ is continuous. 
Proof. Because $C_{I^{\prime}}^{0}(X)$ is itself an inductive limit, it is sufficient to show that the inclusion map

$$
i: C_{k}^{0}(\beta X \backslash Z) \longrightarrow C_{\tau}(X)
$$

is continuous for each zero-set $Z \subset \beta X \backslash X$. Let $Z$ denote the image filter under $i$ of the neighborhood filter at zero in $C_{k}^{0}(\beta X \backslash Z)$. The subset $Z$ can be considered a zero-set of a function $\bar{f}$, where $f$ is a positive function in $C^{0}(X)$. Since $f$ is invertible, we set $g=f^{-1}$ and note that $\mathscr{F}_{1}$ and $\mathscr{F}_{2}$, the Fréchet filters generated by $\left(\frac{1}{n} 1\right)_{n \in N}$ and $\left(\frac{1}{n} g\right)_{n \in N}$, both converge to zero in $C_{\tau}(X)$. Thus, we can find a filter $\theta$ coarser than both $\mathscr{F}_{1}$ and $\mathscr{F}_{2}$, where $\theta$ converges to zero and has a basis $\mathscr{B}$ of solid, $A$-convex sets. To complete the proof, we wish to establish that $\mathscr{U}$ contains $\theta$. Given any $B \in \mathscr{\mathscr { S }}$, clearly, $B \cap C^{0}(X)$ satisfies the conditions of Lemma 2 , and thus, it is sufficient to show that some support set for $B \cap C^{0}(X)$ is contained in $\beta X \backslash Z$. Since $g$ is absorbed by $B$, proceeding as in the proof of Proposition 1, we can choose a scalar multiple of $g$, call it $k$, with the property that $k^{n} \in B$ for every $n \in N$. Since $\bar{g}(Z)=\{\infty\}$, it is clear that the set

$$
G=\{x \in \beta X: \bar{k}(X) \leqq 2\}
$$

is contained in $\beta X \backslash Z$. To establish that $G$ is actually a support set, let $h$ be a bounded function in $C(X)$ with $\bar{h}(G)=\{0\}$. It follows that $|h| \leqq k^{m}$ for some $m \in N$, and thus, $h$ itself is in $B$, since $B$ is solid.

A completely regular topological space $Y$ will be called $z$-realcompact, if every compact subset of $\beta Y \backslash Y$ is contained in a zero-set $Z$, where $Z \subset \beta Y \backslash Y$.

It is evident that any $z$-realcompact space is realcompact, and on the other hand, every locally compact $\sigma$-compact (Hausdorff) topological space is $z$-realcompact (see [3], p. 445 and [5], p. 115).

For a $z$-realcompact space $X$, it can be established by modifying the proof of Theorem 2 in [3] that an ideal in $C_{I^{\prime}}^{\circ}(X)$ is closed, if and only if it is full. (In modifying the proof, the set $K$ would be replaced by a zero-set in $\beta X \backslash X$ containing $K$.) Now, given a closed ideal $J$ in an $A$-convex convergence lattice $C_{\tau}(X)$, where $X$ is $z$-realcompact, Proposition 2 implies that $J \cap C^{0}(X)$ is closed in $C_{I^{\prime}}^{0}(X)$. Thus, $J \cap C^{\circ}(X)$ is a full ideal, and arguing as in the proof of Theorem 1 , we conclude that $J$ itself is a full ideal. To summarize, we state:

THEOREm 4. Let $X$ be a z-realcompact space and $C_{\tau}(X)$ an $A$ convex convergence lattice. Every closed ideal in $C_{\tau}(X)$ is full.

The argument in the proof of the corollary to Theorem 1 is valid for convergence spaces, and thus, we have: 
Corollary. Let $X$ be a z-realcompact topological space and $C_{\tau}(X)$ an A-convex convergence lattice. If $\tau$ restricted to $C^{\circ}(X)$ is finer than the topology of pointwise convergence, then an ideal in $C_{-}(X)$ is closed if and only if it is full.

It is clear that $C_{I^{\prime}}(X)$ and in fact any inductive limit (in the category of convergence spaces) of $m$-convex (respectively, $A$-convex) topological lattices are $m$-convex (respectively, $A$-convex) convergence lattices. The following proposition provides examples of $m$-convex (and hence, $A$-convex) convergence lattices which cannot be realized as inductive limits of topological vector spaces.

For a completely regular topological space $Y$, let $C_{c}(Y)$ denote the algebra $C(Y)$ endowed with the continuous convergence structure (see [1]). In [3] (Theorem 8), it is shown that $C_{c}(Y)$ is not in general an inductive limit of topological vector spaces, while we can prove:

Proposition 3. Let $Y$ be a completely regular topological space. $C_{c}(Y)$ is an m-convex convergence lattice.

Proof. A filter $\theta$ converges to zero in $C_{c}(Y)$ if and only if for every $p \in Y$ and $n \in N$, there exists a neighborhood $U$ of $p$ and a $B$ in $\theta$ such that

$$
B \cdot U \subset\left[\frac{-1}{n}, \frac{1}{n}\right]
$$

(i.e., $|f(x)| \leqq 1 / n$ for every $f \in B$ and $x \in U$ ). Given $\Phi$ convergent to zero in $C_{c}(Y)$, we associate to every point $y \in Y$ and $n \in N$ a neighborhood $U_{(y, n)}$ of $y$ and a $B$ in $\Phi$ so that

$$
B \cdot\left(U_{(y, n)}\right) \subset\left[\frac{-1}{n}, \frac{1}{n}\right] \cdot
$$

Now, we define

$$
T_{(y, n)}=\left\{f \in C(Y): f\left(U_{(y, n)}\right) \subset\left[\frac{-1}{n}, \frac{1}{n}\right]\right\} .
$$

It is clear that all the sets $T_{(y, n)}$ for $y \in Y$ and $n \in N$ generate a filter $\psi$ coarser than $\Phi$ and convergent to zero in $C_{c}(Y)$. Furthermore, it is easy to check that finite intersections of sets $T_{(y, n)}$ are also $m$ convex and solid, which completes the proof.

\section{REFERENCES}

1. E. Binz and H. H. Keller, Funktionenräume in der Kategorie der Limesräume, Ann. Acad. Scie. Fenn. A.I., (1966), 1-21.

2. - On closed ideals in convergence function algebras, Math. Ann., 182 (1969), 145-153. 
3. E. Binz and W. Feldman, On a Marinescu structure on $C(X)$, Comm. Math. Helv., 46 Fasc. 4, (1971), 436-450.

4. A. C. Cochran, E. R. Keown, and C. R. Williams, On a class of topological algebras, Pacific J. Math., 34 (1970).

5. L. Gillman and M. Jerison, Rings of Continuous Functions, Van Nostrand, Princeton, 1960 .

6. E. A. Michael, Locally multiplicatively-convex topological algebras, Amer. Math. Soc. Memoirs, 11 (1952).

7. L. Nachbin, Topological vactor spaces of continuous functions, Proc. N.A.S., 40 (1954), 471-474.

8. Anthony L. Peressini, Ordered Topological Vector Spaces, Harper and Row, New York, 1967.

Received October 16, 1972.

UNIVERSITY OF ARKANSAS 



\section{PACIFIC JOURNAL OF MATHEMATICS}

\section{EDITORS}

RICHARD ARENS (Managing Editor)

University of California

Los Angeles, California 90024

R. A. BeAumont

University of Washington

Seattle, Washington 98105
J. DugundJI*

Department of Mathematics

University of Southern California

Los Angeles, California 90007

D. Gilbarg and J. Milgram

Stanford University

Stanford, California 94305

\section{ASSOCIATE EDITORS}

E. F. BECKENBACH

B. H. NEUMANN

F. WOLF

K. YoSHIDA

\section{SUPPORTING INSTITUTIONS}

UNIVERSITY OF BRITISH COLUMBIA
CALIFORNIA INSTITUTE OF TECHNOLOGY
UNIVERSITY OF CALIFORNIA
MONTANA STATE UNIVERSITY
UNIVERSITY OF NEVADA
NEW MEXICO STATE UNIVERSITY
OREGON STATE UNIVERSITY
UNIVERSITY OF OREGON
OSAKA UNIVERSITY

UNIVERSITY OF BRITISH COLUMBIA CALIFORNIA INSTITUTE OF TECHNOLOGY UNIVERSITY OF CALIFORNIA MONTANA STATE UNIVERSITY NEW MEXICO STATE UNIVERSITY UNIVERSITY OF OREGON OSAKA UNIVERSITY
UNIVERSITY OF SOUTHERN CALIFORNIA STANFORD UNIVERSITY UNIVERSITY OF TOKYO UNIVERSITY OF UTAH WASHINGTON STATE UNIVERSITY UNIVERSITY OF WASHINGTON AMERICAN MATHEMATICAL SOCIETY NAVAL WEAPONS CENTER

* C. R. DePrima California Institute of Technology, Pasadena, CA 91109, will replace J. Dugundji until August 1974. 


\section{Pacific Journal of Mathematics}

\section{Vol. 51, No. $1 \quad$ November, 1974}

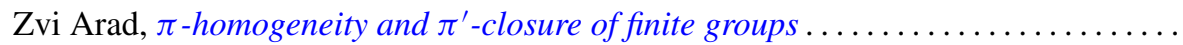

Ivan Baggs, A connected Hausdorff space which is not contained in a maximal

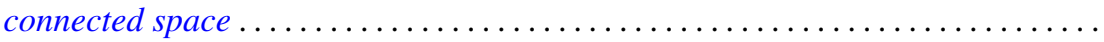

Eric Bedford, The Dirichlet problem for some overdetermined systems on the unit ball in $C^{n}$

R. H. Bing, Woodrow Wilson Bledsoe and R. Daniel Mauldin, Sets generated by

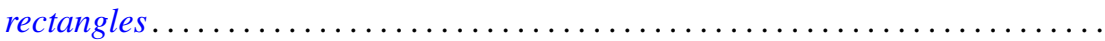

Carlo Cecchini and Alessandro Figà-Talamanca, Projections of uniqueness for

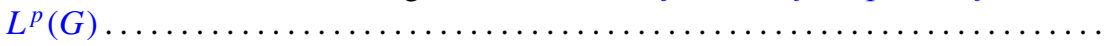

Gokulananda Das and Ram N. Mohapatra, The non absolute Nörlund summability of Fourier series .

Frank Rimi DeMeyer, On separable polynomials over a commutative ring ........ Richard Detmer, Sets which are tame in arcs in $E^{3} \ldots \ldots \ldots \ldots \ldots \ldots \ldots \ldots$

William Erb Dietrich, Ideals in convolution algebras on Abelian groups ..........

Bryce L. Elkins, A Galois theory for linear topological rings .................

William Alan Feldman, A characterization of the topology of compact convergence on $C(X)$.

Hillel Halkin Gershenson, A problem in compact Lie groups and framed cobordism

Samuel R. Gordon, Associators in simple algebras.

Marvin J. Greenberg, Strictly local solutions of Diophantine equations

Jon Craig Helton, Product integrals and inverses in normed rings . . . . . . . . . . . .

Domingo Antonio Herrero, Inner functions under uniform topology . . .

Jerry Alan Johnson, Lipschitz spaces .

Marvin Stanford Keener, Oscillatory solutions and multi-point boundary value

functions for certain nth-order linear ordinary differential equations.

John Cronan Kieffer, A simple proof of the Moy-Perez generalization of the

Shannon-McMillan theorem .......................

Joong Ho Kim, Power invariant rings

Gangaram S. Ladde and V. Lakshmikantham, On flow-invariant sets .

Roger T. Lewis, Oscillation and nonoscillation criteria for some self-adjoint even

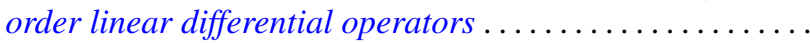

Jürg Thomas Marti, On the existence of support points of solid convex sets ..

John Rowlay Martin, Determining knot types from diagrams of knots . .

James Jerome Metzger, Local ideals in a topological algebra of entire functions

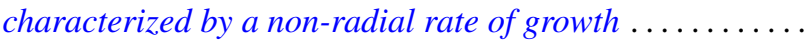

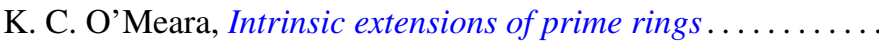

Stanley Poreda, A note on the continuity of best polynomial approximations ..

Robert John Sacker, Asymptotic approach to periodic orbits and local prolongations

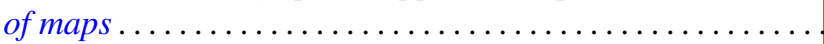

Eric Peter Smith, The Garabedian function of an arbitrary compact set . .

Arne Stray, Pointwise bounded approximation by functions satisfying a side condition

John St. Clair Werth, Jr., Maximal pure subgroups of torsion complete abelian

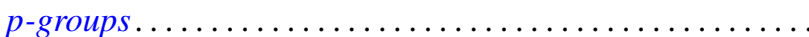

